\title{
Research Paper \\ The Effect of Spiritual Self-care Training on the Suffering of Mothers of Newborns Admitted to the Intensive Care Unit of Sari Hospitals
}

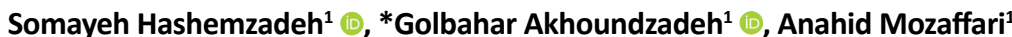

1. Department Nursing, Faculty of Medical Sciences, Aliabad Katoul Branch, Islamic Azad University, Aliabad-e-Katul, Iran.

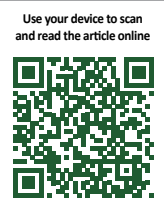

Citaton: Hashemzadeh S, Akhoundzadeh G, Mozaffari A. [The Effect of Spiritual Self-care Training on the Suffering of Mothers of Neonates Admitted to the Respiratory Intensive Care Unit of Hospitals in Sari (Persian)]. Complementary Medicine Journal. 2020; 10(3):284-295. https://doi.org/10.32598/cmja.10.3.1021.1

doishttps://doi.org/10.32598/cmja.10.3.1021.1

Key words:

Spiritual self-care, Caring suffering, Mothers, Intensive care unit, Newborns

\section{ABSTRACT}

Objective Infant hospitalization in the Intensive Care Unit (ICU) may cause many challenges for the family, especially the mother, and cause psychological problems and shock. Self-care, as a prominent factor in physical and mental health and effective in mothers' adaptation to their child's illness. Therefore, this study aimed to investigate the effect of spiritual self-care education on mothers of newborns admitted to the ICU of the hospitals in Sari City.

Methods This experimental study was performed on 64 mothers of newborns hospitalized with informed written consent in the hospitals' respiratory ICU in Sari City in 2019. Sampling was done by an available method and simple random sampling in two groups: Experimental $(n=32)$ and control $(n=32)$. The experimental group received a spiritual self-care training program in 6 sessions of 60 minutes every day. Data were collected using a demographic questionnaire and a 22-item scale of suffering (CBC) and entered into SPSS V. 25 software, then performed using independent statistical tests, Chi-square, Fisher, and ANCOVA (analysis of covariance).

Results The mean of the pre-intervention care range in the experimental and control groups was $65.46 \pm 4.51$ and $62.68 \pm 7.29$, respectively, but the paired t-test did not show a significant difference $(P=0.07)$. According to the paired t-test, the Mean $\pm S D$ care range after the intervention was $43.47 \pm 6.47$ and 60.59 \pm 6.73 , respectively, which led to a significant difference $(P<0.01)$.

Conclusion The results of this study showed that spiritual self-care education reduces maternal care suffering. Therefore, its use is recommended for all caregivers

\section{Extended Abstract}

\section{Introduction}

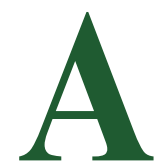

Healthy baby's birth is the best divine gift; however, encountering a premature baby creates a severe psychological crisis for parents. Parents often suffer from a lack of knowledge about the cause and method child's illness, separation from the child, the future of the disease, changes in the patient's physical condition, and other complications. However, in practice, professional caregivers pay more attention to patients' health and are less aware of family experiences and forget about them. Also, people with the lowest level of education tolerate the most suffering from exposure to the disease.

of treatment and care, the economic consequences of the

\section{* Corresponding Author:}

Golbahar Akhoundzadeh, PhD.

Address: Department Nursing, Faculty of Medical Sciences, Aliabad Katoul Branch, Islamic Azad University, Aliabad-e-Katul, Iran.

Tel: +98 (911) 8240624

E-mail: g_akhoundzadeh@aliabadiau.ac.ir 
Religious beliefs, as an activity, help patients reduce their feelings of suffering. As one of the essential aspects of human existence, spirituality has a necessary relationship with people's health. Thus, the spiritual experience can help a person become more adaptable to the stressful conditions of his life and take steps to improve the situation by creating meaning and concept in life and a sense of belonging to God.

Considering the undeniable role of spiritual care in patients' health and the importance of mothers' attitudes in using this care and considering the prevalence of culture and religious principles in child care in Iran, this study investigates the effect of spiritual self-care on mothers' care of newborns. The intensive care unit of Sari hospitals was performed. This study was conducted in intensive care units of the hospitals of Sari City.

\section{Materials and Methods}

This experimental study was performed on 64 mothers of neonates hospitalized with informed written consent in the respiratory intensive care unit of Sari city hospitals in 2019. Sampling was done by the available method and simple random sampling in two experimental groups $(n=32)$ and control $(n=32)$. The experimental group received a spiritual self-care training program in 6 sessions of 60 minutes on alternate days (Session 1: Familiarity with the work process and goals of the group, familiarity with the mothers under study, and establishing communication and mutual trust between the researcher and the mothers. The questionnaires were distributed between both "experimental" and "control" groups, and the parents got to know each other and answer the questionnaire.

They were allowed to talk about their main problems and exchange experiences and information; Session 2: Determining the test and control group by the researcher and getting acquainted with the methods of spiritual self-care with the focus on topics such as trust in God, patience, altruism, and heavenly rewards; Session 3: Familiarity with the concepts of spirituality, spiritual methods, and its effects; Session 4: Familiarity with spiritual practices such as writing diaries, talking with others, reading books, and listening to music; Session 5: Familiarity with sports methods such as hiking and yoga; Session 6: Completion of the questionnaires by both groups, two months after the intervention). Data were collected using a demographic questionnaire and a 22-item suffering scale (CBC) and entered into SPSS V. 25 software, then implemented using independent t-tests, Chisquare, Fisher, and covariance (ANCOVA) statistical tests.

Table 1. Comparison of the suffering of mothers of infants admitted to the neonatal intensive care unit

\begin{tabular}{|c|c|c|c|}
\hline \multirow[b]{2}{*}{ Time } & \multicolumn{2}{|c|}{ Mean $\pm S D$} & \multirow{2}{*}{$\mathbf{P}$} \\
\hline & Control & Experimental & \\
\hline Before intervention & $62.8 \pm 7.29$ & $65.46 \pm 4.51$ & $=0.07$ \\
\hline After intervention & $60.59 \pm 6.76$ & $43.47 \pm 6.47$ & $<0.01$ \\
\hline $\mathrm{P}$ & $\begin{array}{c}<0.01 \\
\text { Mean=2.09 }\end{array}$ & $\begin{array}{c}<0.01 \\
\text { Mean=22.03 }\end{array}$ & \\
\hline
\end{tabular}

Table 2. Effect of spiritual self-care education on the suffering of mothers of neonates admitted to the intensive care unit (ANCOVA test)

\begin{tabular}{ccccccc}
\hline Variables & Total Squares & Degrees of Freedom & Mean Squares & F & Sig. & Eta \\
\hline Modified model & 5450.75 & 2 & 2725 & 87.95 & $\mathrm{P}<0.01$ & 0.73 \\
\hline Post-test separator & 741.38 & 1 & 741.38 & 23.91 & $\mathrm{P}=0.02$ & 0.28 \\
\hline Group & 5530.88 & 1 & 5330.8 & 172.12 & $\mathrm{P}<0.01$ & 074 \\
\hline Error & 1890.21 & 61 & 30.98 & & & \\
\hline Sum & 180501 & 63 & & & & \\
\hline Total & 7340.98 & 64 & & & & \\
\hline
\end{tabular}




\section{Results}

The results showed that $58 \%$ of the neonates were girls, and $42 \%$ were boys. Based on the results, the two experimental and control groups in terms of mother's age $(\mathrm{P}=0.15)$, mother's occupation $(\mathrm{P}=0.30)$, mother's education $(\mathrm{P}=0.15)$, father's occupation $(\mathrm{P}=0.10)$, infant age $(\mathrm{P}=0.06)$, infant $\operatorname{sex}(\mathrm{P}=0.31)$, number of children $(\mathrm{P}=0.80)$, and income $(\mathrm{P}=0.06)$, no statistically significant difference was observed (Table 1).

The Mean $\pm \mathrm{SD}$ of pre-test/post-test scores in the two experimental and control groups are presented in Table 1. Also, by eliminating the effect of the pre-test, the ANCOVA test showed a significant difference in the amount of maternal care suffering between the two groups $(\mathrm{P}<0.01$, $\mathrm{Eta}=0.74)$, which $74 \%$ of post-test changes could be due to the effect of training (Table 2).

\section{Conclusion}

This study showed a significant difference between mothers who received the intervention and mothers who did not receive the intervention in terms of the amount of care suffering. According to the results of this study, the intervention significantly reduced the care suffering of mothers of premature infants in the post-test stage. Comparison of the mean of this variable after the intervention on maternal care suffering showed a significant difference. Thus, by using spiritual self-care, mothers will acquire the ability to withstand many psychological pressures. Also, for mothers affected by spiritual care, by strengthening and activating their spiritual beliefs, their lifestyle takes on a spiritual color.

According to the results of this study, it can be concluded that spiritual care education as an influential factor can reduce the suffering of care in stressful situations such as childhood illness. The limitation of this study was the reluctance of some mothers to participate in the study. Since the above restriction may adversely affect the results' generalizability and limit them, it is suggested that a larger sample size be used in future research.

\section{Ethical Considerations}

Compliance with ethical guidelines

This study was approved by the Ethics Committee of Aliabad Katoul Branch, Islamic Azad University (Code: IR.IAU.CHALUS.REC.1399.003).

\section{Funding}

The present paper was extracted from the MSc. thesis of the first author, Department Nursing, Faculty of Medical Sciences, Aliabad Katoul Branch, Islamic Azad University, Aliabad-e-Katul.

Authors' contributions

Conceptualization, methodology, data collection, data analysis: Sambeh Hashemzadeh; Writing - original draft, and writing - review \& editing, supervision: Golbahar Akhondzadeh; Investigation and methodology, funding acquisition and resources, data analysis: Anahid Mozaffarinia.

\section{Conflicts of interest}

The authors declared no conflict of interest. 


\title{
تأثير آموزش خودمر اقبتى معنوى بر رنج مراقبتى مادران نوزادان بسترى در بخش مراقبتهاى ويزه

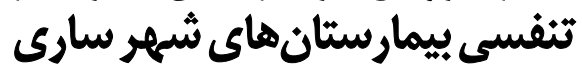

\author{
سميه هاشمزاده' ه. •كلبهار آخوندزاده'ه. اناهيد مظفرىنيا' \\ 1. كروه هرستارى، دانشكده علوم يزشكى، واحد على آباد كتول، دانشكاه آزاد اسلامى، على ابادكتول، ايران.
}

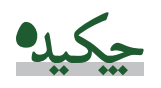

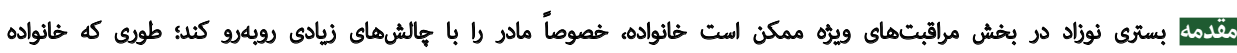

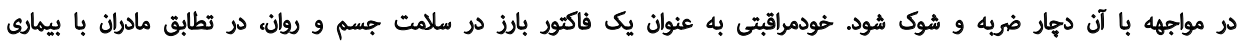

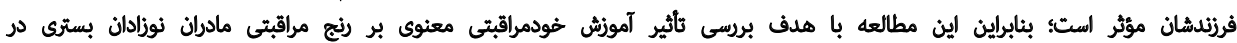

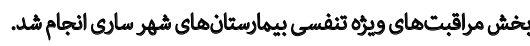

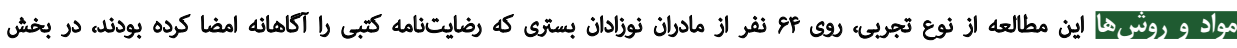

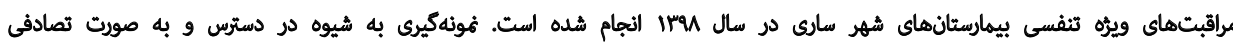

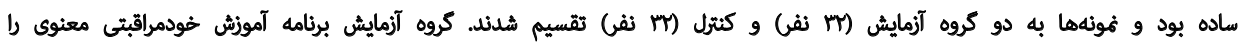

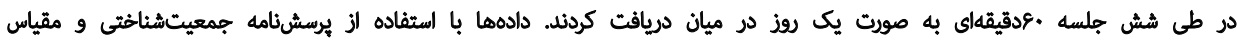

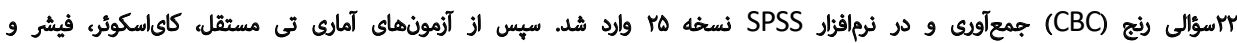
تمليل كوواريانس استفاده شئ.

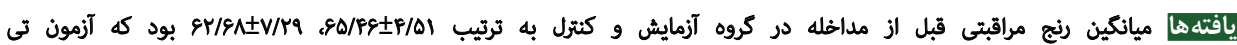

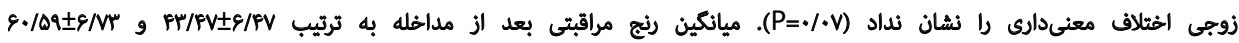

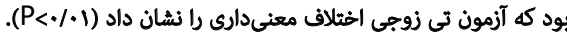

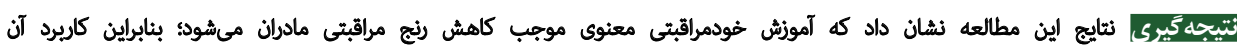
براكى همه مراقبين ييشنهادمى نائود.

\author{
اطلاعات مقاله: \\ تاريخ دريافت: 19 فروردين

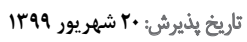

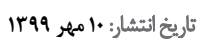

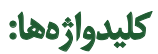

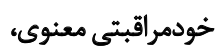

رنج مراقبتى، مادران،

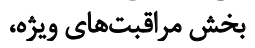

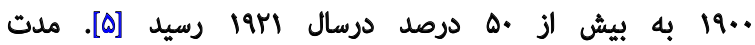

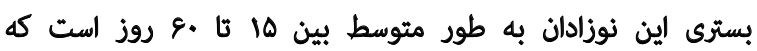

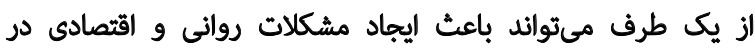

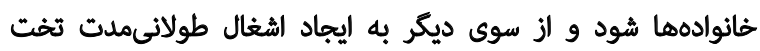

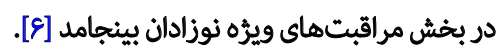

بخش مراقبتهاي ويره نوزادالن،بخشى براي مراقبت از نوزادان

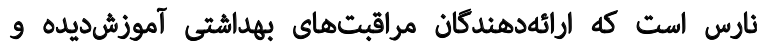

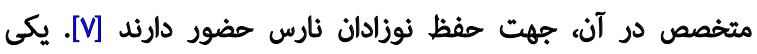

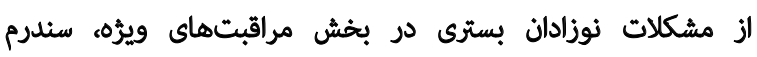

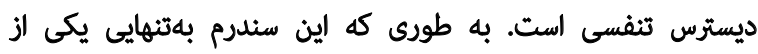

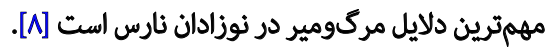
طبق كزارش يونيسف ميزان مركومير نوزادان در جهان الز وب

تولد نوزاد سامل بهترين هديه الهى الست و اين در حالى است

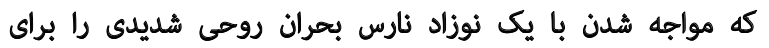
والدين ايجاد مىكند [1]]. طبق تعريف سازمان جهانى بهداشت.

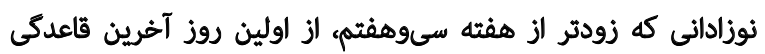

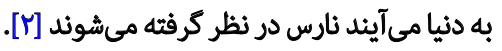
تولد نوزاد نارس به عنوان عامل خطر در •ه درصد مركوميرهاى

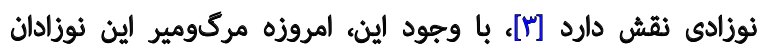

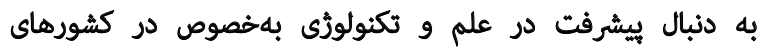

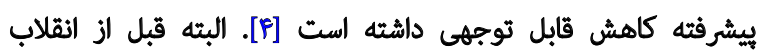

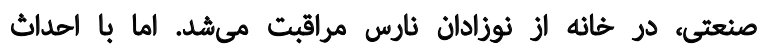

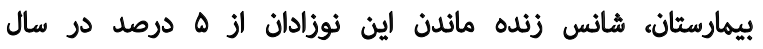

$$
\begin{aligned}
& \text { : نويسئده مسئول: } \\
& \text { دكتر ملبهار آخوندزاده }
\end{aligned}
$$

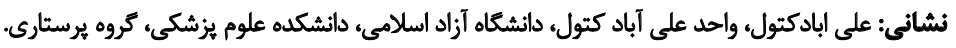

$$
\begin{aligned}
& \text { تلفن: } \\
& \text { g_akhoundzadeh@aliabadiau.ac.ir :يست الكترونيكي }
\end{aligned}
$$


از جنبههاى مهم از وجود آدمى ارتباط مهمى با سلامتى افراد

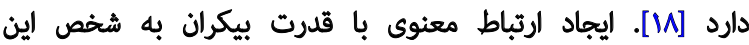

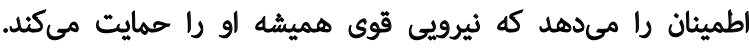

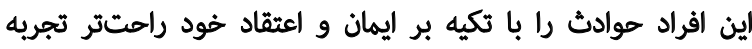

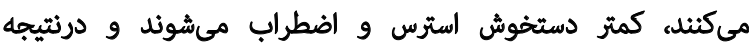

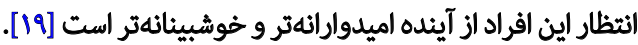

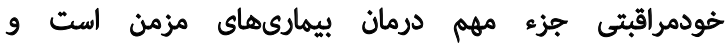

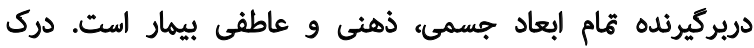

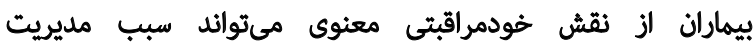

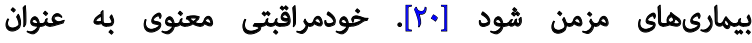

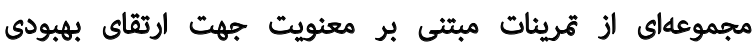

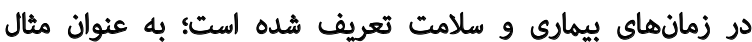

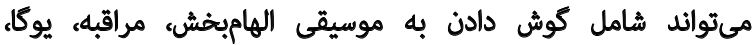

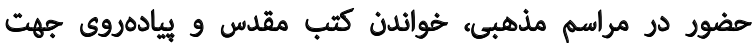

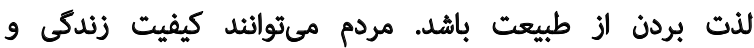

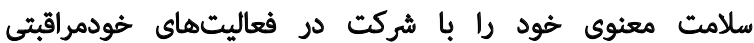

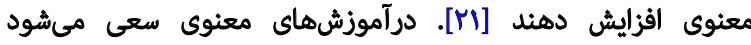

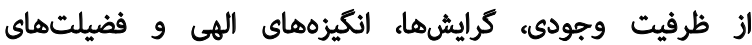

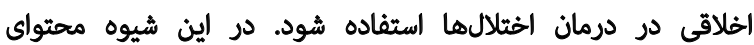

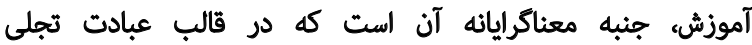

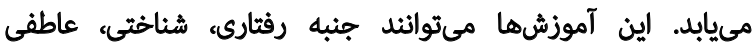

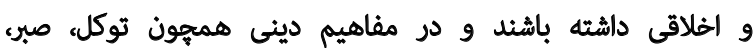

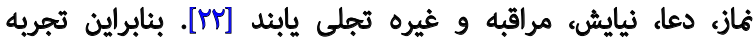

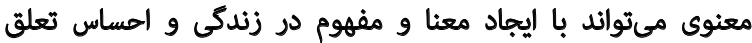

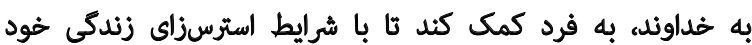

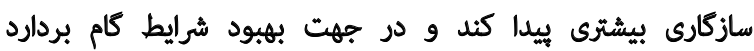

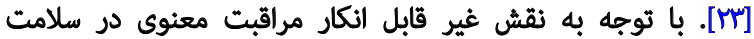

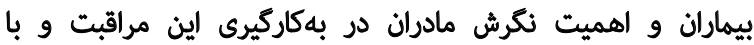

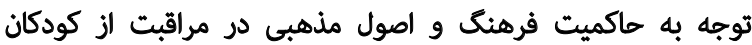

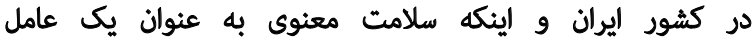

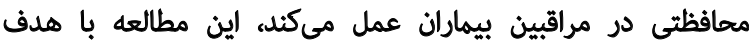

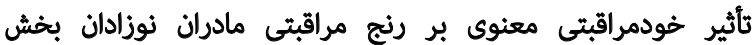
مراقبتهاى ويرٔه تنفسى انجام شدا.

$$
\text { مواد وروشها }
$$

اين مطالعه تجربى با طرح بيش آزمون - يسآزمون و در دو دور

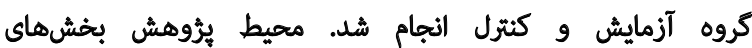

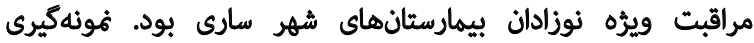

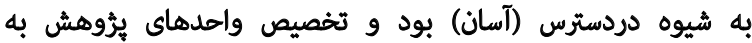

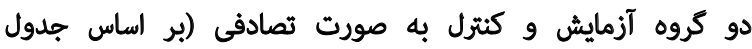

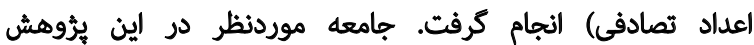

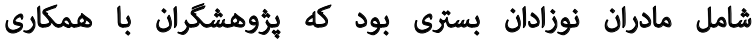

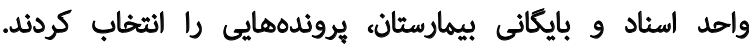

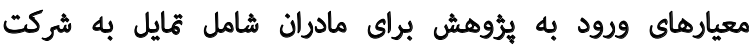

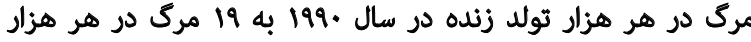

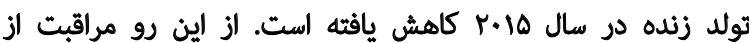

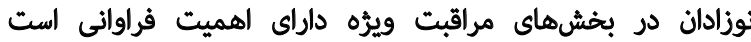

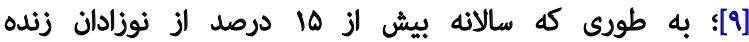

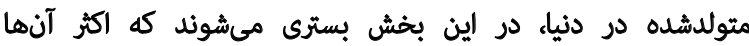

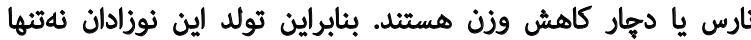

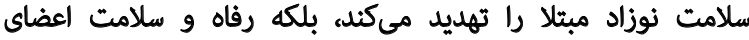

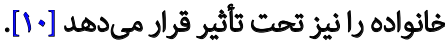

در ميان اعضاى خانواده، مادر لنستين شخصيتى است كه با

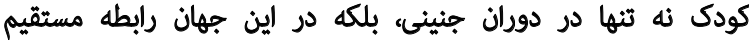

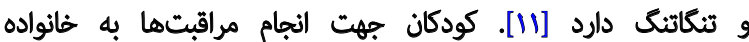

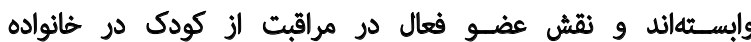

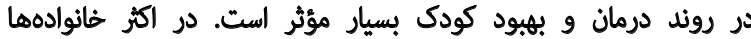

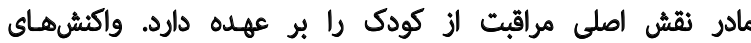

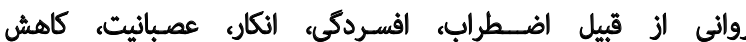

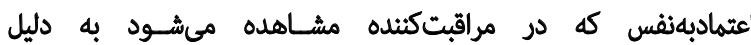
ترس از عود بيمارى و آيثده كودى است. والدين خصوصاً مادين مادران به ديه

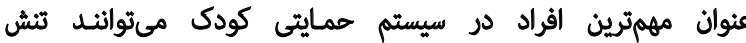

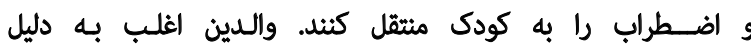

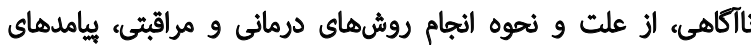

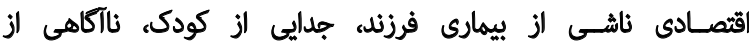

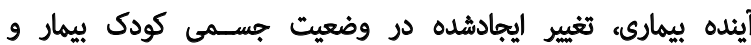

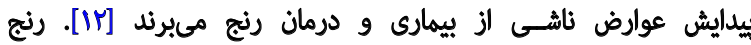

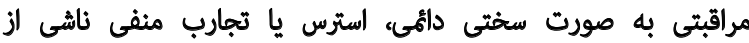

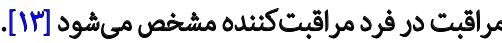

در ارتباط با آثار بيهارى اعثقاد بر اين است كه آنجه انسانها را

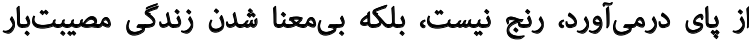

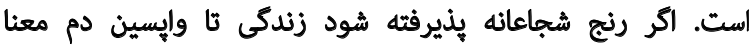

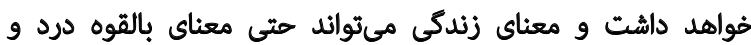

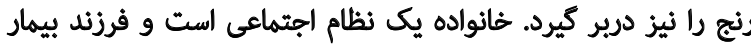

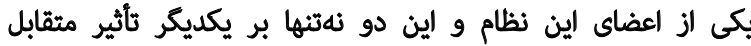

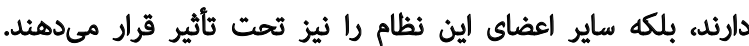

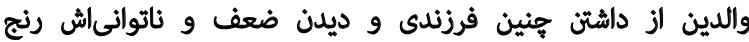

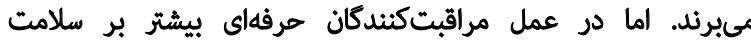

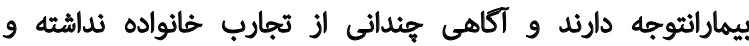

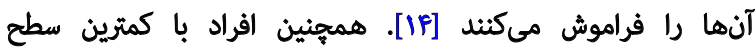

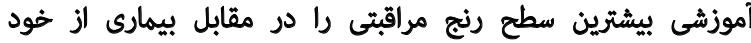

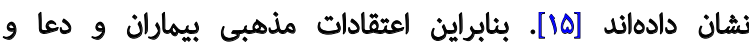

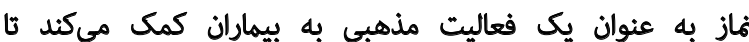

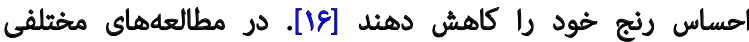

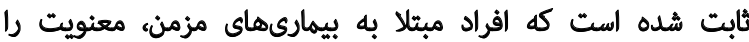

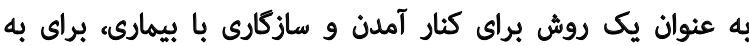

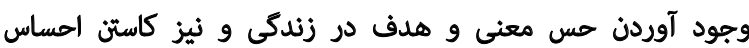

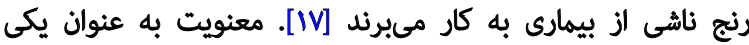


بهندرت، بعضى اوقات و بيشتر اوقات (نمره ا تا F) داده مى أشود.

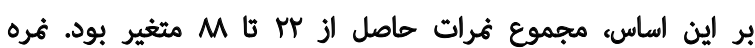

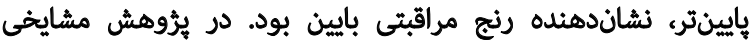

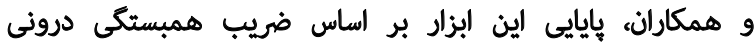

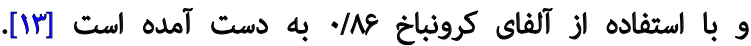
همجنين در مطالعه حاضر، هايايى ابزار نيز توسط آلفاى كرونباخ

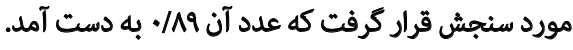

برنامه مداخله براي كروه آزمايش، اموزش توسط يثوهشكر

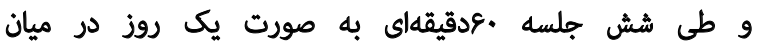

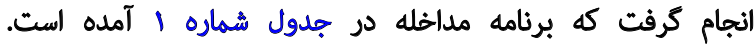

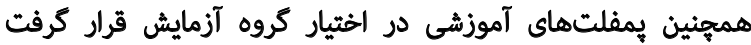

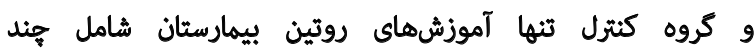
يمفلت حاوى مطالب مختصر درباره بيمارى و مراقبت رون را دريافت

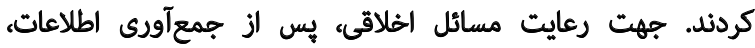
يمفلتهاى آموزشى در اختيار كروه كنترل قرار داده شد.

اين مطالعه توسط كميثه اخلاق دانشكاه آزاد اسلامى واحد جالوس با كد اخلاق تصويب رسيده است. ساير ملاحظات اخلاقى الز جمله رضايت

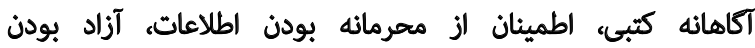

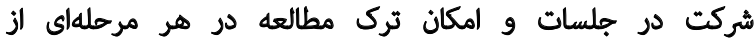

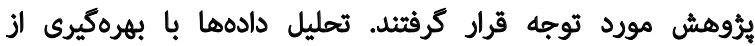

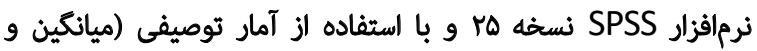

در مطالعه، داشتن حداقل سواد خواندن و نوشتن، مسلمان بودن،

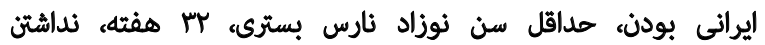

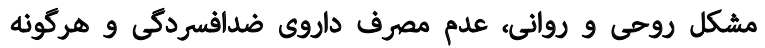

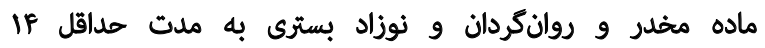

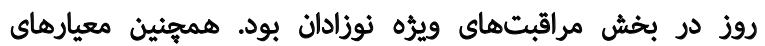

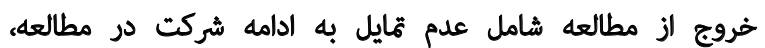

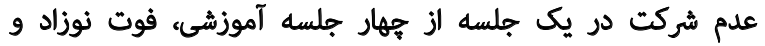

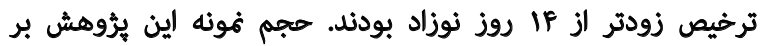

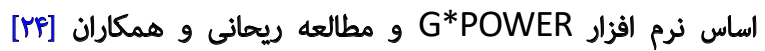

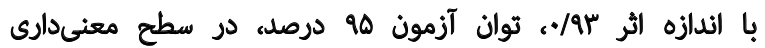

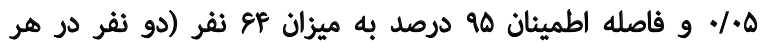
كروه) در نظر كرفته شد. جهت كردآورى دادهها از فرم اطلاعات جمعيتشناختى مادر و

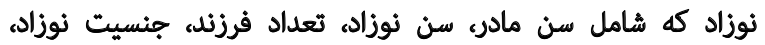

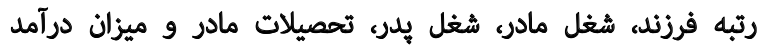

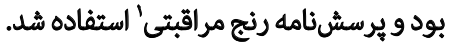
برسشنامه rاسؤالى رنج توسط المستا و همكاران طراحى

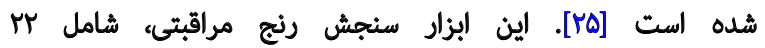

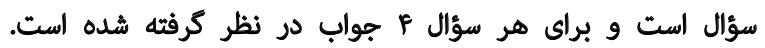
به هر يك الز سؤالات برسشنامه به تروتيب، جوابهائ هركز،

1. Caregiver Burden Scale (CBC)

جدول ا. جلسات آموزشى

محتواي أموزش

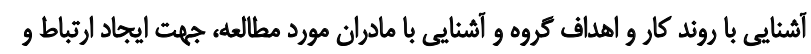

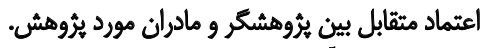

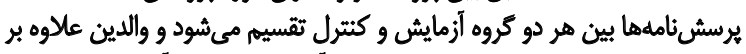

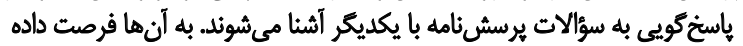

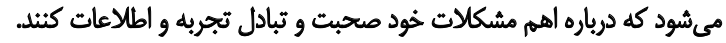

آشنايى با روشهاى خودمراقبتى معنوى با محوريت مباحثى جون توكل، صبر،

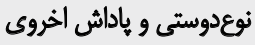

آشنايي با مفاهيهم معنويت، روشهاي معنوى و اثيرات آن

آشنايع با روش هاى هعنوى نظير خاطر هنويسى، صحبت كردن و كتاب خواندن، كوش دادن به موسيقي

أشنايي با روشهاي ورزشي مثل ييادهروى و يوزكا
وقيقه اولين جلسه مدثزتمان دوز جلسات

در إيان جلسه ينجم يكيج أهوزشى شامل كتابهه و CD آهوزشى جهت تثبيت هطالب در اختيار مادران قرار مى كيرد

تكميل برسش نامه ها دو ماه بعد از مداخله توسط دو كروه آزمايش و كنترل دو ماه بعد از مداخله +عدقيقلهاى ششمين جلسه 
در جدول شماره ب ارائه شده است. همهيجين آزمون كوواريانس

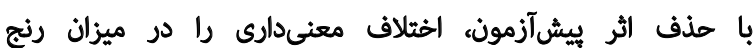

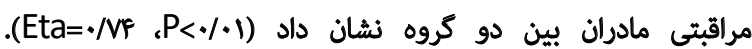

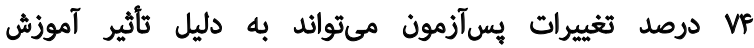
باشيل (جلول شمارهم) بحث با توجه به اهميت رنج مراقبتى، بهويزه براى خانوادههاى
انحراف معيار) و استباطى (آزمونهاى تى مستقل، كاى اسكوثر، فيشر و تحليل كوواريانس) النجام شد. يافتهها

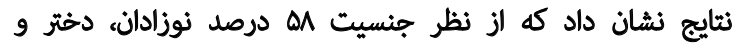

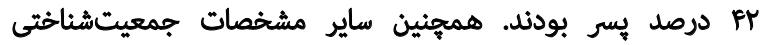
در جدول شماره r آورده شده است. ميانكين و التحراف معين معيار ثمرههاى بيشآزمون و يسآزمون در كروههاى آزمايش و كنترل

جدول r. مقايسه مشخصات جمعيتشئاختى واحدهاى يُؤهش در كروه آزمايش و كنترل

\begin{tabular}{|c|c|c|c|c|}
\hline \multirow{2}{*}{$\mathbf{P}$} & \multicolumn{2}{|c|}{ فراوانى (درصد) } & \multirow{2}{*}{\multicolumn{2}{|c|}{ مشخصات جمعيتشناختى }} \\
\hline & كتترل & 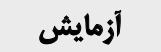 & & \\
\hline \multirow{2}{*}{.$/ M^{*}$} & $r \cdot(\& r)$ & $\operatorname{Ir}(\Delta r)$ & ل ن لختر & \multirow{2}{*}{ جنسيت } \\
\hline & (rN)וr & $10(P V)$ & بـ بسر & \\
\hline \multirow{3}{*}{.$/ \Delta s$} & $9(M V)$ & $r(9)$ & كارمثل & \multirow{3}{*}{ شغل مادر } \\
\hline & $r \cdot(9 Y)$ & $\operatorname{rA}(A V)$ & خانهدار & \\
\hline & $r(q)$ & $1(r)$ & آزاد & \\
\hline \multirow{4}{*}{$\bullet$ ハ** } & $9(\pi \wedge)$ & $\wedge(\Gamma \Delta)$ & 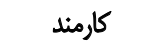 & \multirow{4}{*}{ شغل بلر } \\
\hline & $1 \cdot(r \cdot)$ & $1 \cdot(r \cdot)$ & بيكار & \\
\hline & $r(q)$ & $r(r V)$ & آزاد & \\
\hline & $9(r \Lambda)$ & $r(V)$ & 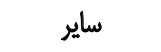 & \\
\hline \multirow{4}{*}{$\cdot / 10 * *$} & $1 \cdot(\mu)$ & $r(q)$ & زير دييلم & \multirow{4}{*}{ سطح تحصيلات } \\
\hline & $r(r v)$ & $10(\$ 9)$ & دييلم & \\
\hline & $V(\pi T)$ & $\operatorname{Ir}(\mathrm{m})$ & كارشناسى & \\
\hline & $r(q)$ & $r(\varepsilon)$ & 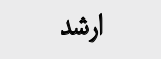 & \\
\hline \multirow{4}{*}{1.900} & $F(I T)$ & $q(T \Lambda)$ & زير Y ميليون & \multirow{4}{*}{ ميزان درآمد } \\
\hline & $\pi(N)$ & $M(\$ \Delta)$ & r-r & \\
\hline & $I(r)$ & $1(r)$ & | ب-8 ميليون & \\
\hline & $P(I T)$ & $1(N)$ & بالاى \& ميليون & \\
\hline.$/ 1 Q^{a+\infty}$ & $r \cdot|\Delta| \pm g / N \mid$ & $Y N Y \pm Y / A Y$ & \multicolumn{2}{|c|}{ سن مادران } \\
\hline .1 .9000 & $\Pi \pi / T V \pm 1 / 1 \Delta$ & $\mathrm{m} / \mathrm{AV} \pm \cdot / \mathrm{q}$ & \multicolumn{2}{|c|}{ سن نوزادان } \\
\hline$\cdot 11^{\cdots \cdots}$ & $1 / \Delta q \pm \cdot / F q$ & $V / \Delta S \pm / \Delta$ & \multicolumn{2}{|c|}{ ت تعداد فرزندان } \\
\hline . & $\mid(A) \pm \cdot / \Delta \Lambda$ & $r / \cdot r \pm V / \cdot r$ & \multicolumn{2}{|c|}{ رتبه فرزند } \\
\hline
\end{tabular}


جدول r. مقايسه ميزان رنج مادران نوزادان بسترى در بخش مراقبتهاي ويره نوزادان

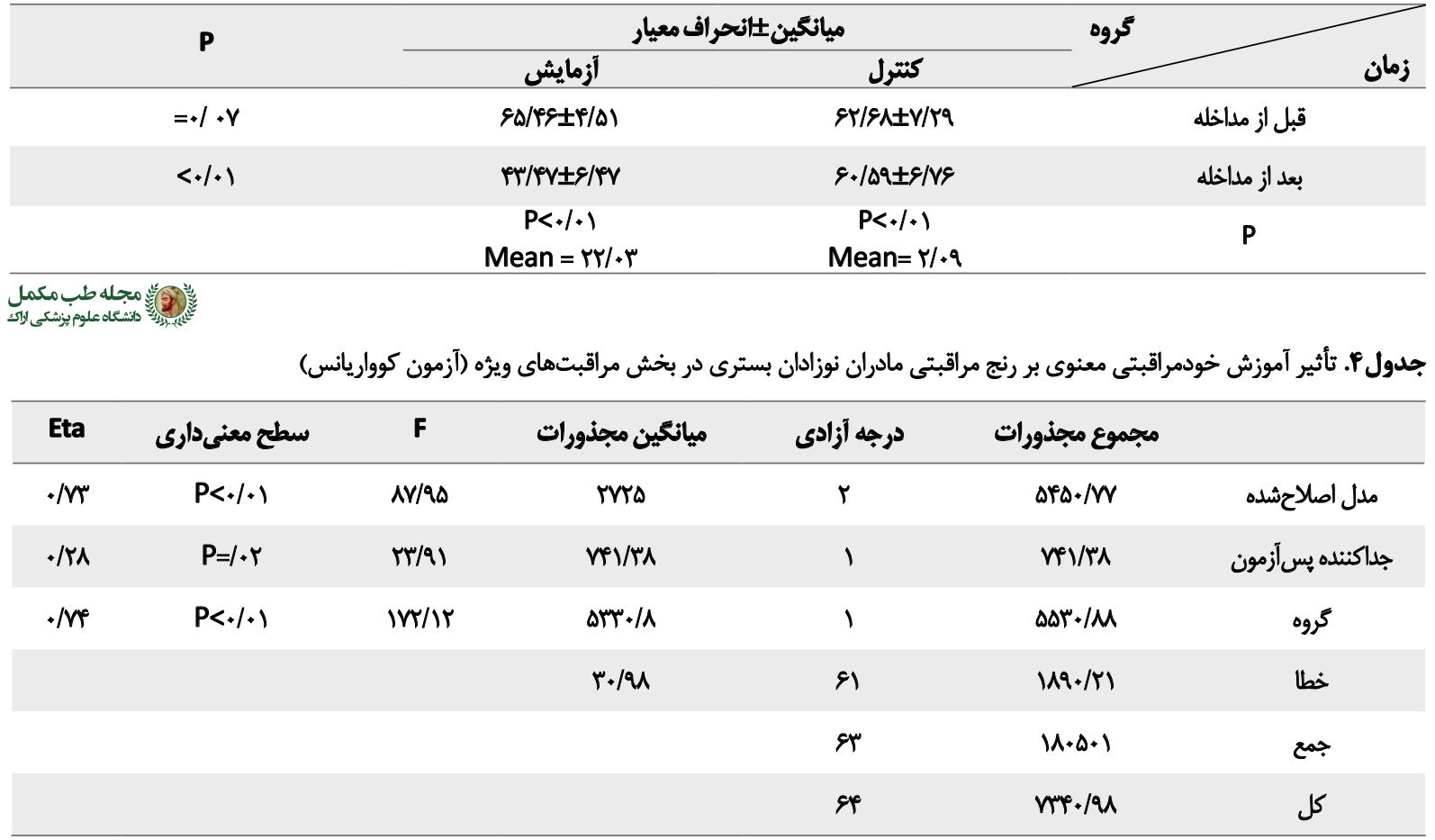

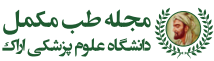

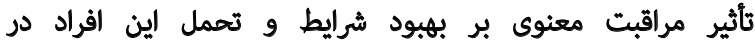

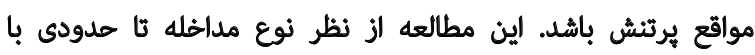

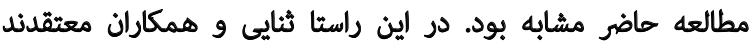

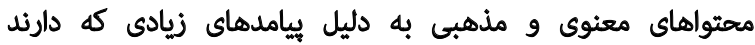

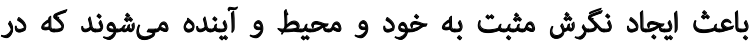

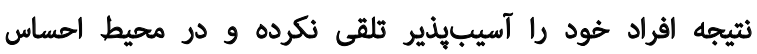

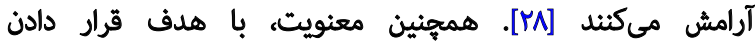
باورهاي فرد به او كمك ميكند ثا وقايع منفى را به شيوهاى بهتر

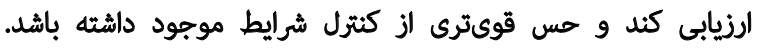

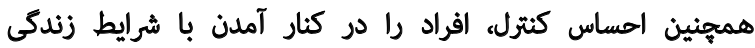

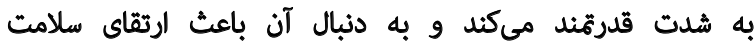

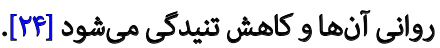

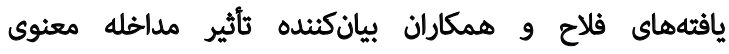
بر كاهش تنش و اضطراب اين افراد بود و درمجمموع سلامت

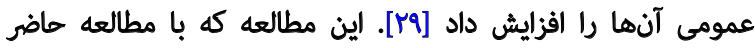

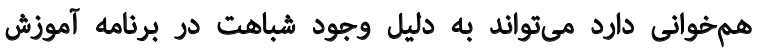

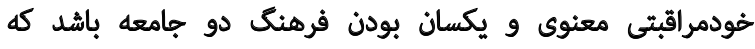

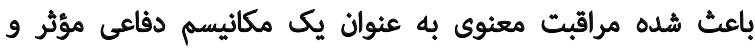

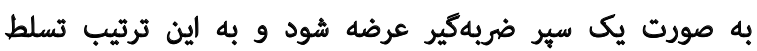

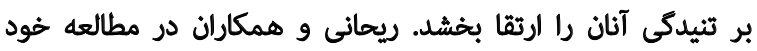

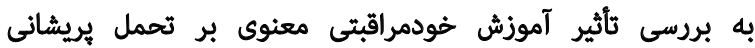

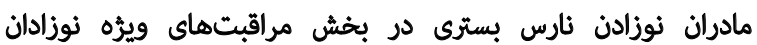

داراى فرزند بيمار، يُروهش حاضر با هدف تعيين ثأثير آموزش

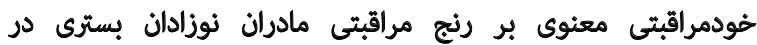

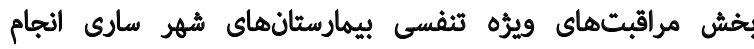

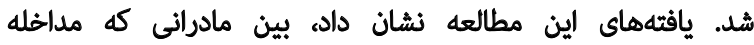

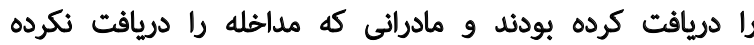

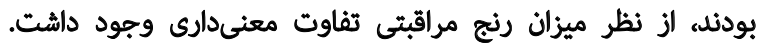

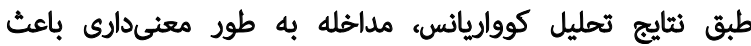

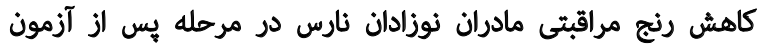

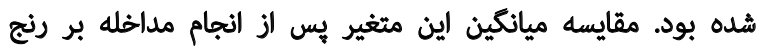

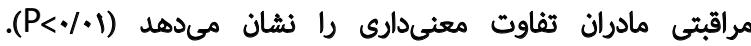

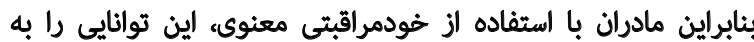

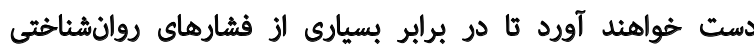

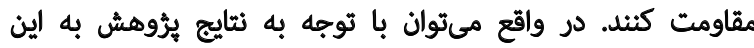

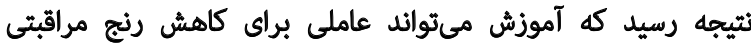
در شرايط استرسزال و يرتش همهيون بيمارى كودكان شود. سخاوتيور و همكاران در يُوهش خود با بردسى ثأثير

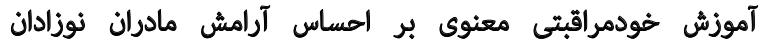

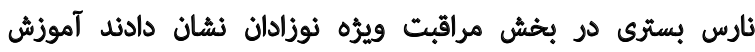

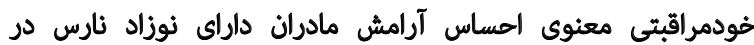

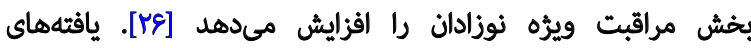

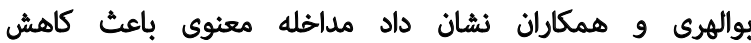

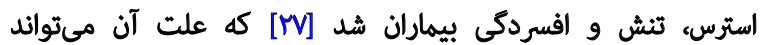


يرداختند. آنها نشان دادند تحمل يريشانى يس از آموزش

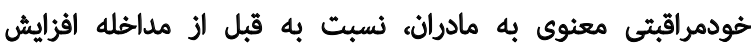
معنى

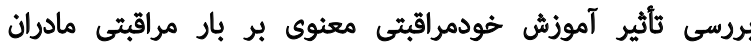

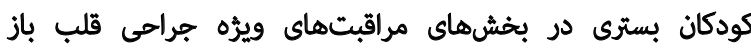

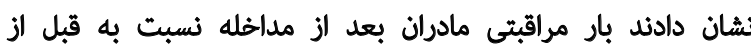

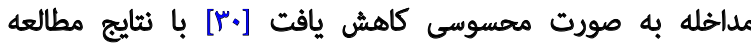
حاضر همسوست.

$$
\text { S) }
$$

بر اساس نتايج حاصل الز اين مطالعه، برنامه مراقبث معنوى.

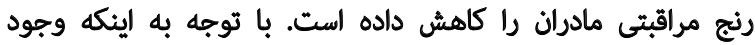

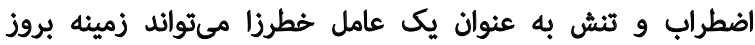

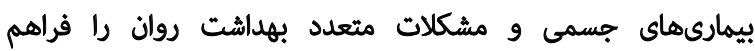

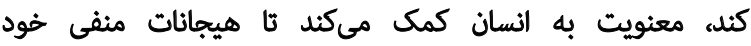

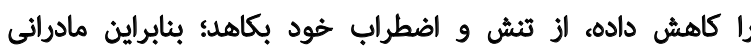

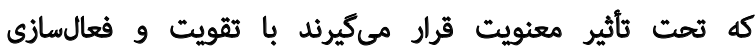

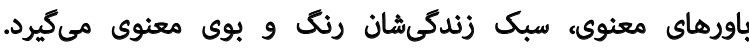

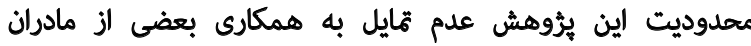

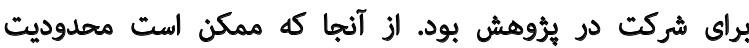

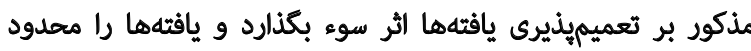

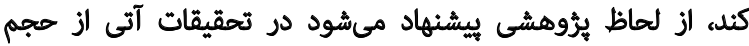

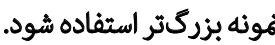

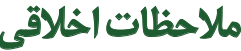

$$
\text { ييروى از اصول اخلاق بئوهش }
$$

IR.IAU.CHALUS.REC.1399.003 اين مقاله با شناسه اخلاق در دانشكاه آزاد اسلامى، واحد على آباد كتول تصويب شده است.

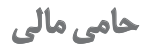

مقاله حافر الز هايان نامه كارشناسى ارشد نويسئده اوله در

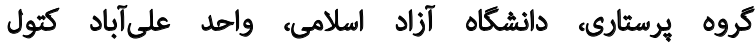
استخراج شده است.

$$
\text { مشاركث نويسندكان }
$$

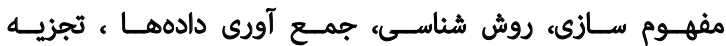

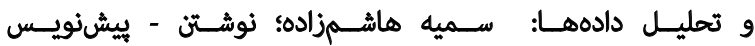

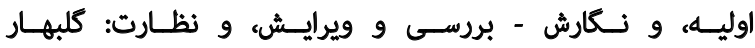

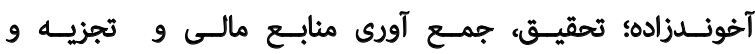

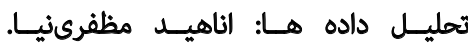

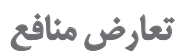

بثابر اظهار نويسندكانء اين مقاله تعارض مثافع ندارد. 


\section{References}

[1] Milan M, Nasimi F. [The effect of family-centered care educational program on anxiety of mothers of premature infants hospitalized in neonatal intensive care unit (Persian)]. Iranian Journal of Nursing Research. 2018; 13(3):49-54. http://ijnr.ir/article-1-2006-fa.html

[2] Zeraati H, Behnam Vashani H, Rezaeian A, Abrishami M, Reihani T, Shoeibi $\mathrm{N}$, et al. [Effect of multisensory stimulation on oxygen saturation in premature infants during eye examination (Persian)]. Evidence Based Care. 2014; 4(4):7-16. [DOI: 10.22038/EBCJ.2014.3655]

[3] Blencowe H, Cousens S, Chou D, Oestergaard M, Say L, Moller A-B, et al. Born too soon: The global epidemiology of 15 million preterm births. Reproductive Health. 2013; 10(Suppl 1):S2. [DOI:10.1186/7424755-10-s1-s2] [PMID] [PMCID]

[4] Lawn JE, Davidge R, Paul VK, von Xylander S, de Graft Johnson J, Costello $A$, et al. Born too soon: Care for the preterm baby. Reproductive Health. 2013; 10(Suppl 1):S5. [DOI:10.1186/742-4755-10-s1-s5.] [PMID] [PMCID]

[5] Zelkowitz P, Feeley N, Shrier I, Stremler R, Westreich R, Dunkley D, et al. The Cues and Care Trial: A randomized controlled trial of an intervention to reduce maternal anxiety and improve developmental outcomes in very low birthweight infants. BMC Pediatrics. 2008; 8:38. [DOI:10.1186/471-2431-8-38.] [PMID] [PMCID]

[6] mokaberian M, Sheikh M, Noripour S, Kashani Vm. [Effects of one period of tactile kinetic stimulations of preterm neonates on state anxiety of their mothers (Persian)]. Koomesh. 2016; 17(4):821-8. http:// koomeshjournal.semums.ac.ir/article-1-2774-en.html

[7] Brandão S, Figueiredo B. Fathers' emotional involvement with the neonate: Impact of the umbilical cord cutting experience. Journal of Advanced Nursing. 2012; 68(12):2730-9. [DOI:10.1111/j.3652648.012.05978.x.] [PMID]

[8] Mansoori M, Janani S, Chavoshi D, Mohaghegh P, Hemmatpour S, Fatolahpour A, et al. [Evaluation of the Side Effects and mortality of Surfactant therapy with mechanical ventilation comprise with manual ventilation in Neonates Admitted to NICU ward of Besat hospital of Sannandaj in 2012-2013 (Persian)]. Razi Journal of Medical Sciences. 2017; 24(155):66-72. http://rjms.iums.ac.ir/article-1-4054-en.html

[9] Ghavami SR, Borimnejad L, Seyedfatemi N, Haghani H. [The effect of parental role training on stress in the parents of hospitalized newborns in a neonatal intensive care unit (Persian)]. Journal of Hayat. 2017; 23(3):243-53. http://hayat.tums.ac.ir/article-1-2002-en.html

[10] Mirjalili M, Azizzade forouzi M, Sabzevari S, heidarzadeh A, Haghdost $A$. [Assess the admission needs of the mothers of hospitalized in the neonatal intensive care unit (Persian)]. Journal of Pediatric Nursing. 2015; 2(2):1-9. http://jpen.ir/article-1-128-en.html

[11] Hoseini Ghomi T, Salimi Bajestani H. [Effectiveness of resilience training on stress of mothers whose children, suffer from cancer in imam khomeini hospital of Tehran (Persian)]. Journal of Health Psychology. 2012; 1(4):97-109. http://hpj.journals.pnu.ac.ir/article_444 en.html

[12] Elahi Asgar Abad H, Behnam Vashani H, Heshmati Nabavi F, Badiei Z. [The effect of caregivers on the children with cancer under going chemotherapy to prevention gastrointestinal side effects of chemotherapy (Persian)]. The Journal of Urmia Nursing and Midwifery Faculty. 2016; 14(1):39-46. http://unmf.umsu.ac.ir/article-1-2583-en.html

[13] Mashayekhi F, Rfati Sh, Rfati F, Pilehvarzadeh M, Mohamadi Sardo MR. [Assessment of caregiver burden in mothers of children with major thalassemia in Jiroft city (Persian)]. Modern Care Journal. 2014; 11(3):229-35. https://www.sid.ir/en/Journal/ViewPaper. aspx?ID=435736
[14] Pouraboli B, Abedi H, Abbaszadeh A, Kazemi M. [Silent Screams: Experiences of caregiver suffering by parents of children with thalassemia: A qualitative study (Persian)]. Journal of Qualitative Research in Health Sciences. 2014; 3(3):281-91. https://www.sid.ir/en/journal/ ViewPaper.aspx?id=430302

[15] Adeosun II. Correlates of caregiver burden among family members of patients with schizophrenia in lagos, Nigeria. Schizophrenia Research and Treatment. 2013; 2013:353809. [DOI:10.1155/2013/.] [PMID] [PMCID]

[16] Pirasteh Motlagh AK, Nikmanesh Z. [The relationship of spirituality with the pain and quality of life in patients with HIV/AIDS (Persian)] Armaghane-Danesh. 2012; 17(4):337-48. http://armaghanj.yums. ac.ir/article-1-247-en.html

[17] Krause N, Bastida E. Religion, suffering, and health among older Mexican Americans. Journal of Aging Studies. 2009; 23(2):114-23. [DOI:10.1016/j.jaging.2008.11.002] [PMID] [PMCID]

[18] Bagheri F, Akbarzadeh F, Hatami $H$. The relationship between nurses' spiritual intelligence and happiness in Iran. Procedia - Social and Behavioral Sciences. 2010; 5:1556-61. [DOI:10.1016/j.sbspro.2010.07.325

[19] Asgari P, Roushani K, Mohri AM. [The Relationship between religious belief, optimism and spiritual well being among college students of Islamic Azad University. (Persian)]. Journal of Social Psychology (New Findings in Psychology). 2009; 4(10):27-39. https://www.sid.ir/en/ journal/ViewPaper.aspx?id=194385

[20] Salimi T, Tavangar H, Shokripour S, Ashrafi H. [The effect of spiritual self-care group therapy on life expectancy in patients with coronary artery disease: An educational trial (Persian)]. Journal of Rafsanjan University of Medical Sciences. 2017; 15(10):917-28. http://journal. rums.ac.ir/article-1-3337-en.htm

[21] White ML. Spirituality self-care practices as a mediator between quality of life and depression. Religions. 2016; 7(5):54. [DOI:10.3390/ rel7050054.]

[22] Iri Z, Hojjati H, Akhoundzadeh G. [The impact of spiritual self- care education on self- efficacy in adolescents with thalassemia in children's taleghani hospital of Gonbadkavoos, Golestan 2017-2018 (Persian)]. Iranian Journal of Nursing Research. 2019; 14(1):8-13. http:// ijnr.ir/article-1-2177-en.html

[23] Valikhani A, Yarmohammadi-Vasel M. [The relationship between attachment styles and death anxiety among cardiovascular patients (Persian)]. Journal of Kerman University of Medical Sciences. 2014 21(4):355-67. http://jkmu.kmu.ac.ir/article_16205.htm

[24] Rihani T, Sekhavatpour Z, Heydarzadeh M, Mousavi SM, Mazloom SR. [Investigating the effects of spiritual self-care training on psychological stress of mothers with preterm infants admitted in neonatal intensive care unit (Persian)]. Iranian Journal of Obstetrics, Gynecology and Infertility 2014; 17(97):18-28. [DOI:10.22038/IJOGI.2014.2826]

[25] Elmståhl S, Malmberg B, Annerstedt L. Caregiver's burden of patients 3 years after stroke assessed by a novel caregiver burden scale. Archives of Physical Medicine and Rehabilitation. 1996; 77(2):177-82 [DOI:10.1016/s0003-9993(96)90164-1]

[26] Reyhani T, Sekhavat Pour Z, Heidarzadeh M, Mousavi SM, Mazloom SR. [Investigating the effects of spiritual self-care training on psychological stress of mothers with preterm infants admitted in neonatal intensive care unit (Persian)]. The Iranian Journal of Obstetrics, Gynecology and Infertility. 2014; 17(97):18-27. [DOI:10.22038/ ijogi.2014.2826] 
[27] Bolahri G, Nazari GH, Zamaniyan S. [Effective therapeutic spirituality to reduce the amount of intellectual depression, anxiety and stress in women with breast cancer (Persian)]. Sociol of Women. 2012; 3(9):85-115. http://jzvj.miau.ac.ir/article_1177_en.html

[28] Sanaei B, Nasiri H. [The effect of cognitive-spritual group therapy in reducing depression and anxiety in patients with mood disorders in Isfahan Noor Medical Center (Persian)]. Counseling Research and Development. 2011; 2(7-8):89-97.

[29] Fallah R, Gilzari M, Dstani M, Zahirodin A, Mosavi SM, Smaeeli Akbari $M$. [Effectiveness of spiritual intervention on increasing hope and mental health of women suffering from breast cancer (Persian)]. Journal of Thought and Behavior in Clinical Psychology. 2011; 5(19):65-76. https:// www.sid.ir/en/journal/ViewPaper.aspx?id=208295

[30] Dalir M, Mashouf S, Esmailpourzanjani S. [The effect of spiritual selfcare education on the care burden of mothers with children hospitalized in intensive care units for open heart surgery (Persian)]. Complementary Medicine Journal. 2020; 10(1):34-45. [DOI:10.32598/cmja.10.1.866.1.] 
This Page Intentionally Left Blank 